\title{
Comparison of Various Guidance Strategies to Achieve Time Constraints in Optimal Descents
}

\author{
Ramon Dalmau* and Xavier Prats ${ }^{\dagger}$ \\ Technical University of Catalonia (UPC), 08860, Castelldefels, Spain \\ Ronald Verhoeven, ${ }^{\ddagger}$ Frank Bussink ${ }^{\S}$ and Bart Heesbeen ${ }^{\text {II }}$ \\ Netherlands Aerospace Centre (NLR), 1059 CM, Amsterdam, The Netherlands
}

\section{Nomenclature}

$\begin{array}{ll}D & =\text { aerodynamic drag, } \mathrm{N} \\ E_{k} & =\text { kinetic energy, } \mathrm{J} \\ E_{p} & =\text { potential energy, } \mathrm{J} \\ E_{S} & =\text { specific energy, } \mathrm{m} \\ E_{t} & =\text { total energy, } \mathrm{J} \\ f & =\text { fuel flow, } \mathrm{kg} / \mathrm{s} \\ f & =\text { dynamics of the system } \\ g & =\text { gravity acceleration, } \mathrm{m} / \mathrm{s} \\ \boldsymbol{g} & =\text { path constraints } \\ h & =\text { altitude, } \mathrm{m} \\ K_{\beta} & =\text { penalty for the speed brakes deployment, } \mathrm{kg} / \mathrm{s} \\ J & =\text { cost function } \\ L & =\text { aerodynamic lift, } \mathrm{N} \\ \mathcal{L} & =\text { Lagrange term of the cost function } \\ m & =\text { aircraft mass, } \mathrm{kg} \\ M & =\text { Mach number } \\ M_{M O} & =\text { maximum operational Mach } \\ n & =\text { load factor } \\ \boldsymbol{p} & =\text { parameters vector } \\ s & =\text { along path distance, } \mathrm{m} \\ & \end{array}$

*PhD Student, Department of Physics, C/Esteve Terradas 5, Building C3, Office 121

†Associate Professor, Department of Physics, C/Esteve Terradas 5, Building C3, Office 104

\} \text { Senior Researcher, Cockpit and Flight Operations Division, P.O. Box } 9 0 5 0 2 .

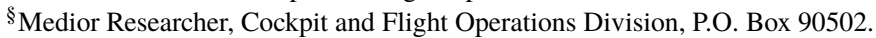

"ISenior Researcher, Cockpit and Flight Operations Division, P.O. Box 90502. 


$$
\begin{array}{ll}
T & =\text { engine thrust, } \mathrm{N} \\
u & =\text { control vector } \\
v & =\text { true airspeed, } \mathrm{m} / \mathrm{s} \\
v_{C A S} & \text { calibrated airspeed, } \mathrm{m} / \mathrm{s} \\
v_{C A S, \max } & =\text { maximum operational calibrated airspeed, } \mathrm{m} / \mathrm{s} \\
v_{C A S, S} & =\text { target speed when the aircraft is in configuration } 1, \mathrm{~m} / \mathrm{s} \\
W_{S} & =\text { longitudinal wind speed, } \mathrm{m} / \mathrm{s} \\
W_{x} & =\text { cross wind speed, } \mathrm{m} / \mathrm{s} \\
\boldsymbol{x} & =\text { state vector } \\
\beta & =\text { speedbrakes status } \\
\gamma & =\text { throttle setting } \\
\pi & =\text { Mayer term of the cost function } \\
\phi &
\end{array}
$$

Subscripts

$\begin{array}{ll}0 & =\text { initial } \\ f & =\text { final }\end{array}$

\section{Introduction}

Continuous Descent Operations (CDOs) have been subject of extensive research in the last decades. Even if proving successful in reducing the environmental impact [1], such operations suffer from a well-known drawback: the loss of predictability from the Air Traffic Control (ATC) point of view, in terms of overfly-times at certain fixes along the route. Consequently, existing CDO implementations require ATC to introduce additional sequencing buffers to ensure safe separation among aircraft, thus reducing airport capacity. For all these reasons, in busy airports, CDOs are only feasible in off-peak hours, when the traffic demand is low [2].

In other to face this issue, several Air Traffic Management (ATM) concepts have been proposed that aim to enable CDOs also in high traffic demand scenarios. For instance, several works investigated the use of fixed Fight-Path-Angle (FPA) descents with time control to improve both spatial and temporal predictability [3, 4]. The disadvantage of fixed FPA descents is that predictability is achieved at the cost of thrust settings that might be different from idle.

Another potential approach to enable CDOs in dense traffic scenarios consists of assigning controlled times of arrival (CTAs) to each aircraft at some strategic fixes for separation, sequencing and merging tasks. This concept of flight operations assumes a negotiation process via data-link between the ATC and the on-board Flight Management 
Systems (FMSs), typically before the Top Of Descent (TOD). This negotiation starts with the on-board computation of the earliest and latest achievable times of arrival at the metering fix, and the subsequent down-link of this information to the ground automation system. Based on this feasible time window and the surrounding traffic a CTA is computed by a ground-based support tool, such as an arrival manager. Then, the incoming CTA is entered on-board as a Required Time of Arrival (RTA) into the FMS, and the on-board trajectory planner computes a new (optimal) trajectory plan starting at the current state, while satisfying the RTA and other operational constraints (e.g., altitude and speed constraints) [5].

Because of modeling errors and uncertainties encountered during the execution of the descent plan (e.g., inaccuracies in the weather forecast or aircraft performance models used by the FMS), aircraft may deviate from this planned altitude and/or speed profile and may also be delayed or advanced with respect to the planned time schedule. Various guidance strategies can be used to safely execute the descent and accurately comply with RTAs.

At present, however, 4D capabilities are only available in the most modern FMSs, which essentially predict periodically the Estimated Time of Arrival (ETA) at the target metering fix to determine the deviation between the RTA and the ETA. If this deviation exceeds a pre-defined threshold, the cruise/descent speed schedule is modified by varying the Cost Index (CI) such that the ETA equals the RTA [6, 7]. A different strategy was proposed in Ref. [8], which continuously adapts the geometric path to nullify time deviations and, only if necessary, modifies the speed plan.

Moreover, some FMSs use the elevator control surface to follow the speed plan (speed-on-elevator) while maintaining idle thrust [9]. In order to follow the speed plan, however, the aircraft might deviate from the planned path. Excessive altitude deviations are corrected by applying thrust or deploying speed brakes [10]. Other more basic FMSs use a different strategy, which consists of using the elevator to follow the planned path (path-on-elevator) while maintaining idle thrust. If required, thrust is added or speed brakes are deployed when the speed deviation exceeds a certain threshold.

An excellent review of state-of-the-art guidance strategies designed to comply with RTAs can be found in Ref. [11]; while Ref. [9] investigated and compared the impact on predictability of the executed trajectory for different guidance strategies, showing that the speed-on-elevator provides the most accurate temporal predictability at a metering fix on the descent trajectory, at the cost of a reduced vertical predictability for the remainder of the descent and increased fuel burn.

Despite modern FMSs are able to comply with RTAs at the destination runway within 15 seconds [12], the speed and/or path adjustments used to correct time deviations are not explicitly optimized for fuel consumption. This motivates the need of new guidance strategies able to compute, on-board and in real-time, optimal altitude and/or speed adjustments to comply with the RTA in presence of uncertainties in the most environmentally friendly way.

A set of additional guidance strategies have been proposed with this aim as a part of the Time and Energy Managed Operations (TEMO) concept [13, 14]. These methods have in common to manage the total energy of the aircraft (altitude and speed) such that time constraints are satisfied while minimizing throttle and speed brakes actions. For instance, if the aircraft is late with respect the RTA and at the same time has an excessive potential energy, altitude could be exchanged by speed with the elevator to satisfy the RTA at no extra cost. 
To the best of our knowledge, a comprehensive comparison of guidance strategies to satisfy RTAs during a CDO has not been performed before. This note evaluates the performances of four guidance strategies: tactical, strategic, hybrid and Model Predictive Control (MPC), which working principles will be presented in Section II.B. The comparison is focused on the environmental impact of these guidance strategies, in terms of noise and fuel consumption, along with their ability to satisfy operational constraints, including RTA compliance. Several descents are generated introducing different weather forecast and aircraft performance modeling errors in a high-fidelity, fast-time flight simulator.

\section{Background}

FMSs suitable for time-constrained CDOs require a trajectory planner capable of generating, in real-time, a (optimal) plan satisfying one or several RTAs along with typical operational constraints. Moreover, a guidance component is also needed in order to execute this trajectory plan and accurately fulfill the RTAs.

\section{A. Optimal trajectory planning}

The TEMO trajectory planner formulates the optimization of the aircraft trajectory as an optimal control problem, which goal is to find the optimal time history of the control vector $\boldsymbol{u} \in \mathbb{R}^{n_{u}}$ and the time-independent parameters $\boldsymbol{p} \in \mathbb{R}^{n_{p}}$ of a system with a state vector $\boldsymbol{x} \in \mathbb{R}^{n_{x}}$ such that a cost function defined over an either free or fixed time interval $\left[t_{0}, t_{f}\right]$ is minimized [15] subject to several constraints:

$$
\begin{array}{ll}
\min _{\boldsymbol{u}(t)} & J:=\phi\left(\boldsymbol{x}\left(t_{f}\right), \boldsymbol{p}\right)+\int_{t_{0}}^{t_{f}} \mathcal{L}(\boldsymbol{x}(t), \boldsymbol{u}(t), \boldsymbol{p}) \\
\text { s.t } & \boldsymbol{x}\left(t_{0}\right)=\boldsymbol{x}_{0} \\
& \dot{\boldsymbol{x}}=\boldsymbol{f}(\boldsymbol{x}(t), \boldsymbol{u}(t), \boldsymbol{p}) \\
& \boldsymbol{g}(\boldsymbol{x}(t), \boldsymbol{u}(t), \boldsymbol{p}) \geq 0 \\
& \boldsymbol{\psi}\left(\boldsymbol{x}\left(t_{f}\right), \boldsymbol{p}\right)=0
\end{array}
$$

The optimal control problem consists of a cost function $J \in \mathbb{R}^{n_{x}} \times \mathbb{R}^{n_{u}} \times \mathbb{R}^{n_{p}} \rightarrow \mathbb{R}$ composed by a running cost or Lagrange term $\mathcal{L} \in \mathbb{R}^{n_{x}} \times \mathbb{R}^{n_{u}} \times \mathbb{R}^{n_{p}} \rightarrow \mathbb{R}$, an end cost or Mayer term $\phi \in \mathbb{R}^{n_{x}} \times \mathbb{R}^{n_{p}} \rightarrow \mathbb{R}$ as well as dynamic constraints $f \in \mathbb{R}^{n_{x}} \times \mathbb{R}^{n_{u}} \times \mathbb{R}^{n_{p}} \rightarrow \mathbb{R}^{n_{x}}$ in the form of Ordinary Differential Equations (ODEs) with initial conditions $\boldsymbol{x}_{0} \in \mathbb{R}^{n_{x}}$, path constraints $\boldsymbol{g} \in \mathbb{R}^{n_{x}} \times \mathbb{R}^{n_{u}} \times \mathbb{R}^{n_{p}} \rightarrow \mathbb{R}^{n_{g}}$ and terminal constraints $\boldsymbol{\psi} \in \mathbb{R}^{n_{x}} \times \mathbb{R}^{n_{p}} \rightarrow \mathbb{R}^{n_{\psi}}$.

In this note, the state vector $\boldsymbol{x}=[v, \gamma, h, s, m]$ is composed by the True Airspeed (TAS), the aerodynamic flight-path angle, the geometric altitude, the distance to go and the mass of the aircraft; and the control vector $\boldsymbol{u}=[\pi, \beta, n]$ is composed, respectively, by the throttle setting, the speed brakes status (either retracted or deployed) and the load factor ${ }^{*}$ In addition, time-independent parameters have not been considered (i.e., $\boldsymbol{p}=[]$ ). The expressions for the cost function,

\footnotetext{
*The load factor is defined as $n=L / m g$, where $L$ is the aerodynamic lift force and $g$ is the local gravity acceleration.
} 
dynamics of the state vector and constraints will be particularized in Section III

An optimal control problem can be defined over more than one phase, where the dynamics of the state vector, the terminal and/or path constraints might change. For multi-phase problems, a new set of constraints is added to link certain state variables across two consecutive phases, in order to guarantee some continuity in the solution [16].

There are several ways to solve the optimal control problem described by Eq. (1). In this note, direct collocation methods have been used. Such direct collocation methods transform the original continuous (and thus infinite) optimal control problem into a (discrete and finite) Non-Linear Programming (NLP) problem [16]. In this way, the time histories of control and state variables are discretized at a set of collocation points, being the differential equations $f$ approximated by some continuous function (such as polynomials) over each collocation step. The values of these discretized variables, along with $\boldsymbol{p}$ (if any), become the unknowns of the new finite variable problem, which can be formulated as a NLP problem and solved by commercial off-the-shelf solvers.

Ideally, the NLP optimization problem should be solved instantaneously, i.e., without delay. In practical situations, however, the execution time is difficult to predict. Despite the execution time for the implementation presented in this note is typically lower than 5 seconds, in some particular conditions it may be relatively high, leading to potential stability issues and degrading the performance for real-time applications. Yet, several techniques can be used to save computational time and improve convergence. In this note, the warm-start technique has been used, which consists of initializing the variables of the NLP optimization problem with a shifted guess from the previously computed optimal solution.

\section{B. Time and energy managed guidance strategies}

The total energy of the aircraft is composed by the sum of its kinetic and potential energy. The total energy of an aircraft can be increased by applying engine thrust, and decreased by increasing the aerodynamic drag. In addition, the law of conservation of energy states that potential energy can be exchanged for kinetic energy and vice versa through energy modulation. It is well-known that throttle and speed brakes are the most effective means to increase and decrease the total energy, respectively, whereas elevator control provides an effective mean to modulate this energy.

The core principle of the TEMO concept is that the energy of the aircraft can be managed in such a way that the RTAs are always satisfied: speed-on-elevator is used to exchange potential energy (altitude) and kinetic energy (speed) at an appropriate rate, while throttle and speed brake are only applied when energy needs to be added or removed from/to the system, respectively. A TEMO descent plan, computed by solving Eq. (1), can be executed using several guidance variants, depending on how time and energy errors are managed. These variants are described below. 


\section{Tactical guidance}

The deviations from the planned time at the current distance to go are continuously nullified by using speed-onelevator, while the throttle and the speed brakes act together to maintain the planned energy level. Using this strategy, the aircraft is continuously following the initially computed descent plan with minimum energy and time deviations, thus there is no need to update the plan during the course of the descent, unless reaching the operational limits of the guidance laws. However, this logic typically has a negative impact on the environment (and on engine wear and tear) because the continuous usage of thrust (and frequent usage of speed brakes) to nullify these energy deviations.

\section{Strategic re-planning}

Using this guidance strategy both Calibrated Airspeed (CAS) and thrust plans are followed. On the one hand, the CAS plan is followed using the elevator (speed-on-elevator); on the other hand, the thrust plan (typically at idle) is followed acting on the throttle. Different from the tactical strategy, time and energy deviations are allowed as long as they do not exceed a predefined threshold. Otherwise, the trajectory predictor optimizes again the trajectory, enforcing the initial conditions $\boldsymbol{x}_{0}$ to the current state and time. This results in a new CAS and thrust plan that minimizes fuel consumption and speed brakes use while satisfying all the constraints (including RTAs).

An advantage of the strategic guidance is that the entire remaining trajectory is taken into account when correcting deviations. In addition, not continuously correcting time and energy deviations could lead to positive effects if the errors caused by different sources of uncertainty are counteractive. However, one must be cautious when selecting the energy and time error thresholds, a critical task that is not straightforward. If the thresholds are too permissive, it could be too late to react and satisfy all applicable constraints when reaching the time and or energy error threshold.

In a previous work [17], this guidance technique was compared with the guidance implementation on a typical FMS that does not correct time errors, showing superior performances in terms of fuel consumption and RTA compliance.

\section{Hybrid guidance}

This strategy is obtained as a result of combining the tactical and the strategic guidances described above. On the one hand, a tactical controller nullifies sustained time errors using speed-on-elevator. On the other hand, the throttle executes the thrust plan (typically at idle). Eventual energy deviations caused by uncertainties and inaccuracies in the descent plan are corrected by means of strategic re-planning whenever the pre-defined threshold is exceeded. As a result, the aircraft is no longer affected by time errors, at the expense of being more prone to energy deviations.

\section{Model Predictive Control (MPC)}

Using this guidance strategy, the remaining descent trajectory is regularly optimized starting at the current aircraft state, without waiting for energy and time deviations to exceed a certain threshold. Then, the resulting CAS and thrust 
plans are executed using the elevator and throttle, respectively, only until the next re-plan instant. Then, the process is repeated moving forward the time horizon [18].

MPC could be seen as the limit of the strategic guidance approach with energy and time deviation thresholds tending to zero, in which the optimal plan is updated in a tactical way. Other research [19] has recently demonstrated the feasibility of using MPC to achieve precise spacing between aircraft, the objective of interval management operations.

\section{Set up of the experiment}

This note compares the performance of the four guidance strategies presented in Section II.B The comparison has been performed for a realistic scenario, and the effects of weather forecast and aircraft performance modeling errors have been investigated through a batch study composed by 492 simulations. Fuel consumption, time and energy deviations at a metering fix, the number of speed brakes deployments, and the area affected by the aircraft noise have been selected as metrics for this comparison. Aircraft noise contours have been calculated using the model described in [20].

\section{A. Scenario}

The experiment has been performed simulating descents to Barcelona-El Prat airport (Spain), using four Standard Terminal Arrival Routes (STARs) published for runway 25L. These STARs, which are shown in Fig. 1. end at one of the four Initial Approach Fixes (IAF) and then, all the approaches finally merge into a single intermediate fix (SOTIL). Later on, the Instrumental Landing System (ILS) glide slope is intercepted 4 NM later (at the final approach point). For this note, SOTIL (the intermediate fix) has been selected as the metering fix where RTA will be assigned.

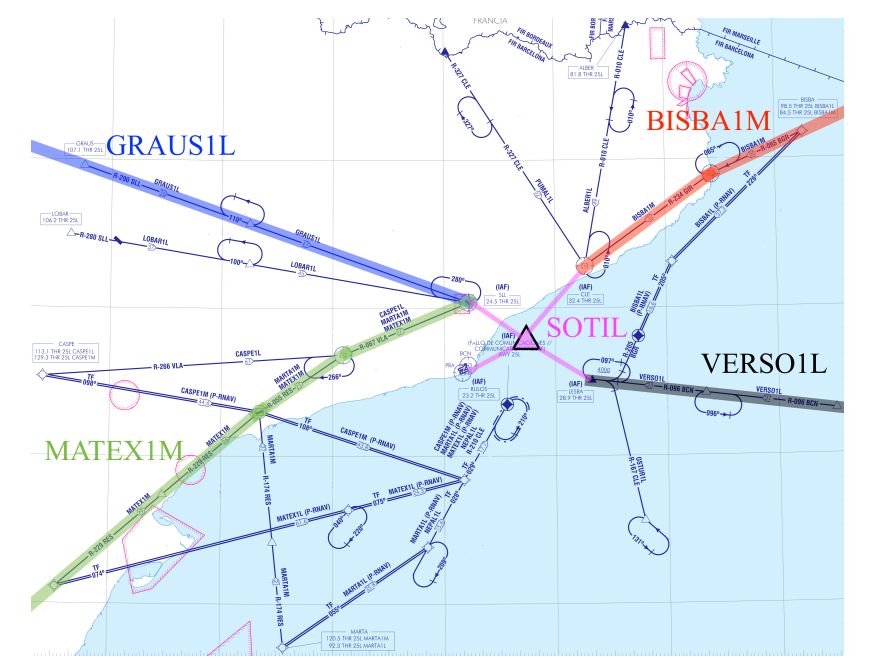

Fig. 1 STARs for runway 25L of Barcelona-El Prat airport

An Airbus A320 model has been used for the experiment. It should be noted that even if both flight simulator and TEMO trajectory predictor draw upon the same aircraft type, the dynamics and performance model used by these components are different: on the one hand, the flight simulator uses a high fidelity six-degrees-of-freedom (6DoF) 
model to represent the aircraft dynamics, and very accurate look-up tables obtained from experimental tests for the aircraft performance. On the other hand, the trajectory predictor represents the aircraft as a three-degrees-of-freedom (3DoF) point-mass model, and approximates the aircraft performance tables with polynomial functions.

Immediately after initiating a simulation, a control time of arrival at SOTIL is received from an hypothetical ATC, and a re-plan is triggered to generate an optimal descent plan satisfying the entered RTA at this waypoint.

Three different RTAs have been considered in this note, enforcing to arrive at SOTIL after 21, 22 and 23 minutes from the start of the simulation, respectively. It should be noted that for this particular scenario, the earliest energy-neutral trajectory ${ }^{\dagger}$ in International Standard Atmosphere (ISA) conditions, calm winds and nominal aircraft performance models could bring the aircraft to the metering fix in 19 minutes, and the latest energy-neutral trajectory in 27 minutes.

Right after configuring with flaps at the green dot speed the pseudo-pilot implemented in the fast-time simulator presses the approach button (this is achieved few nautical miles before SOTIL), disabling the TEMO logic and engaging the ILS guidance modes. Consequently, all the simulations end when SOTIL is reached and all the metrics used for the comparison (fuel consumption, time deviation, energy error, etc.) are computed at this metering fix.

\section{B. Formulation of the trajectory optimization problem}

The trajectory predictor of the TEMO trajectory management concept generates the optimal descent plan subject to RTAs by solving a constrained non-linear optimal control problem, as described in Section II.A.

All simulations start at a distance to go of 140 NM from the landing runway threshold, cruising at FL360 and Mach 0.78, and with a mass corresponding to the $90 \%$ of the Maximum Landing Mass (MLM). Therefore, the initial conditions of the optimal control problem for the first trajectory plan are:

$$
\boldsymbol{x}_{0}=\left[\left.v\right|_{M=0.78}, 0, \mathrm{FL} 360,140 \mathrm{NM}, 90 \% \text { of } \mathrm{MLM}\right]^{T}
$$

The TEMO trajectory predictor expresses the dynamics of the aircraft with the following point-mass model:

$$
\boldsymbol{f}=\left[\begin{array}{c}
\dot{v} \\
\dot{\gamma} \\
\dot{h} \\
\dot{s}
\end{array}\right]=\left[\begin{array}{c}
\frac{T(v, h, \pi)-D(v, h, m, \beta)}{m}-g \sin \gamma \\
\frac{g}{v}(n-\cos \gamma) \\
v \sin \gamma \\
\sqrt{v^{2} \cos ^{2} \gamma-W_{x}(h, s)^{2}}+W_{s}(h, s) \\
-f(v, h, \pi)
\end{array}\right]
$$

where $T$ is the total thrust force; $D$ is the aerodynamic drag force; $f$ is the total fuel flow; and $W_{x}$ and $W_{s}$ are, respectively,

\footnotetext{
${ }^{\dagger}$ An energy-neutral trajectory is performed at idle thrust and without using speed brakes all along the descent. In other words, it does not add nor remove energy to the system by means of throttle or speed brakes actions, respectively.

${ }^{\ddagger}$ Green dot speed is the minimum operating speed in managed mode and clean configuration, being approximately the best lift-to-drag ratio speed.
} 
the cross and along path wind components. In this model, the effects of the bank angle in the aerodynamic drag, the vertical wind component and the wind speed derivatives have been neglected, aiming to reduce the complexity of the mathematical model and, consequently, the computational burden of the NLP optimization problem.

Aiming at reducing the environmental impact, the cost function includes fuel consumption and speed brakes use:

$$
\mathcal{L}=f(v, h, \pi)+K_{\beta} \beta
$$

where $K_{\beta}$ is a weighting factor that determines the penalty associated to the speed brakes deployment, if compared to the fuel flow. In this note, $K_{\beta}=1$. Furthermore, path constraints are specified as follows:

$$
\boldsymbol{g}=\left[\begin{array}{c}
v_{C A S}(v, h)-v_{C A S, S} \\
v_{C A S, \text { max }}-v_{C A S}(v, h) \\
M_{M O}-M(v, h) \\
\pi-0 \\
1-\pi \\
\beta-0 \\
1-\beta \\
1.15-n \\
n-0.85
\end{array}\right] \geq\left[\begin{array}{l}
0 \\
0 \\
0 \\
0 \\
0 \\
0 \\
0 \\
0 \\
0
\end{array}\right]
$$

where $\pi=0$ and $\pi=1$ correspond to idle and maximum thrust settings, respectively; $\beta$ is a binary variable that can only take two levels: either retracted (0) or deployed (1); bounds on the load factor have been chosen to provide good passengers comfort; and $v_{C A S, \max }$ and $M_{M O}$ are, respectively, the maximum operational calibrated airspeed ( $\left.v_{C A S}\right)$ and Mach number $(M)$.

The trajectory plan ends at SOTIL ( $s_{\text {SOTIL }}$, at $v_{C A S, S}$ 多 and at 3,000 ft, being ready to transition to the next flaps setting, intercept the ILS glide path and land. Therefore, the terminal constraints are:

$$
\psi=\left[\begin{array}{c}
s-s_{\text {SOTIL }} \\
v_{C A S}(v, h)-v_{C A S, S} \\
h-3,000 \mathrm{ft}
\end{array}\right]=\left[\begin{array}{l}
0 \\
0 \\
0
\end{array}\right]
$$

\footnotetext{
${ }^{\S} v_{C A S, S}$ is the target speed when the aircraft is in configuration 1.
} 


\section{Configuration of the guidance strategies}

The tactical guidance logic has been configured to continuously adjust the commanded CAS such that time deviations are nullified using speed-on-elevator. Regarding the specific energy (defined as the total energy divided by the weight of the aircraft), a maximum allowed deviation of $50 \mathrm{ft}$ has been set. In the case of being above this threshold, speed brakes are deployed; in the case of falling below, the throttle acts to recover the planned energy level by commanding small amounts of thrust. It is important to remark that under no circumstances the trajectory will be updated during the execution of the descent. This strategy is similar to those used by modern FMSs with RTA function.

The specific energy deviation bounds for the strategic and hybrid guidances have been set to $500 \mathrm{ft}$ in the cruise phase until the TOD and $100 \mathrm{ft}$ at the metering fix, while time deviation bounds for the strategic guidance have been set to 10 and 3 seconds at these two points, respectively. Between these two points, specific energy and time deviation bounds were linearly interpolated. Finally, the MPC has been configured to update the plan every 30 seconds, regardless of the present energy and time deviations. This strategy is activated at the beginning of the descent, aiming to avoid a continuous shift of the TOD.

\section{Weather forecast and performance modeling errors}

The trajectory predictor has been configured to compute the descent plan assuming ISA conditions, calm winds and nominal aircraft performance models (i.e., those corresponding to the flight simulator). It should be noted, however, that the optimization problem presented in Section III.B is generic enough to consider realistic temperature and wind data by approximating them with continuous and twice-differentiable functions of the altitude and/or distance to go. In this note, the high-fidelity flight simulator is configured with wind and/or non-standard atmospheric conditions in order to generate mismatches between the trajectory prediction (computed assuming ISA and calm winds) and the reality. Similarly, the aircraft performance models of the high-fidelity flight simulator have been modified by adding variations with respect to those used by the trajectory predictor of the TEMO algorithm.

\section{Weather forecast errors}

The accuracy of the plan computed by the trajectory predictor, and especially the computation of the ETA over the metering fix, critically depends on the quality of the weather forecast. Wind and temperature accuracy requirements for the future air traffic management system have been assessed before. According to [21], standard deviations of $5 \mathrm{kt}$ and $2.5 \mathrm{~K}$ for the along track wind and temperature, respectively, were considered as a requirement to compute accurate trajectory predictions. In this note, positive and negative temperature errors of 5 and $10^{\circ} \mathrm{C}$ and wind speed errors of 5 and $10 \mathrm{kt}$ have been considered, while pressure errors are left for future work.

It is important to remember that the trajectory predictor computes the plan considering ISA and calm winds. As a result, the temperature errors are translated to ISA deviations during the execution of the simulation. Similarly, wind 
speed errors are accomplished by simply adding winds that were not considered during the planning process.

The two wind speeds chosen for this experiment have been combined with the four classical compass wind directions, leading to a total of eight simulations with wind errors for each combination of guidance strategy, lateral route and CTA. Regarding the temperature errors, a single STAR has been considered, because results are independent of the route, provided that the ISA deviation is not a function of the distance to go.

\section{Aircraft performance modeling errors}

Inaccuracies in the aircraft performance models are also a non-depreciable source of error when predicting trajectories. These models are composed by a set of parameterized functions or look-up tables that express aircraft characteristics (e.g., drag coefficient) as a function of certain variables (e.g., lift coefficient and Mach).

In this note the effects of errors in the drag coefficient and idle thrust models have been analyzed. These aircraftrelated performance functions are expected to have the highest impact on the accuracy of the predicted trajectory. Overestimations and underestimations of 5\% in the magnitude of these two aircraft features have been considered in this experiment. Therefore, a total of four simulations with aircraft performance model errors have been executed for each combination of CTA and guidance strategy. As for the case with temperature errors, a single STAR has been considered for the analysis, because results are independent of the lateral route.

\section{Results}

This section shows the working principles of the four guidance strategies presented in Section II.B by means of an illustrative example. Then, the results of the batch study are discussed.

\section{A. Illustrative example}

The simulation chosen for this illustrative example corresponds to that executed by following the BISBA1M STAR (see Fig.11, subject to a RTA requiring to arrive 23 minutes after the start of the simulation, with approximately $10 \mathrm{kt}$ of unexpected head wind (because the route is not straight and the wind from the east is not completely aligned with the track of the aircraft), in ISA conditions, and without aircraft performance modeling errors.

Figure 2 shows, for each guidance strategy, the time and specific energy deviations ( $\Delta t$ and $\Delta E_{s}$, respectively) along with their thresholds (if any), as a function of the distance to go. Figures 3 and 4 show the planned and executed states and controls for the different guidance strategies, respectively.

According to Fig.2(a) and 4(a) in tactical guidance the time error caused by the unexpected head wind is continuously nullified by using speed-on-elevator, while energy deviations are corrected by either deploying speed brakes or adding minimum amounts of thrust whenever these deviations exceed $50 \mathrm{ft}$. Figure 3(a) illustrates that using this mechanism the initial plan computed before the TOD is never updated. Figure 4(a) shows that the throttle acted twelve times to 


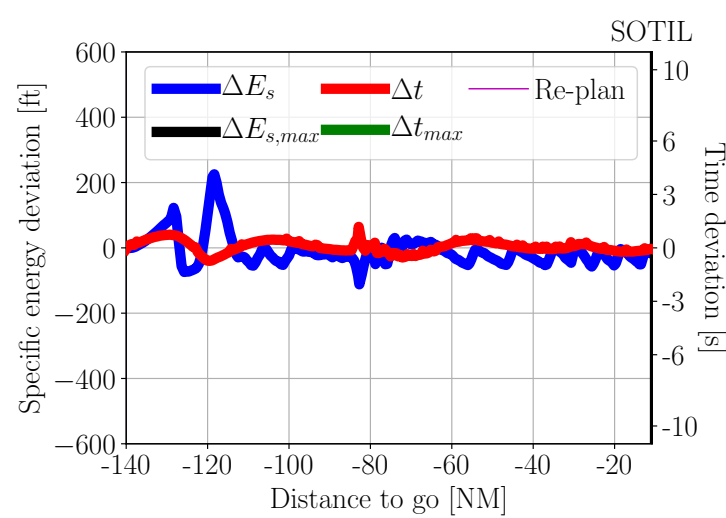

(a) Tactical

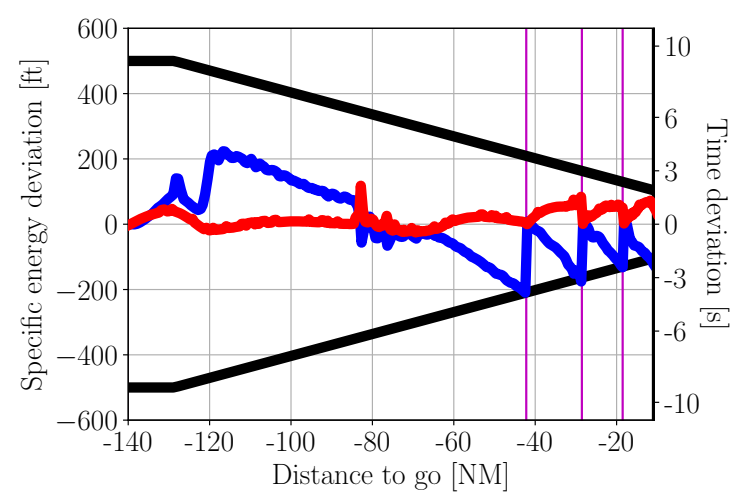

(c) Hybrid

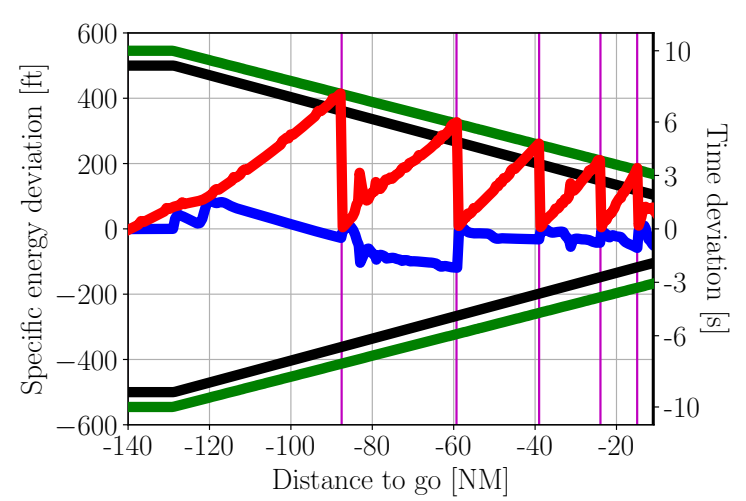

(b) Strategic

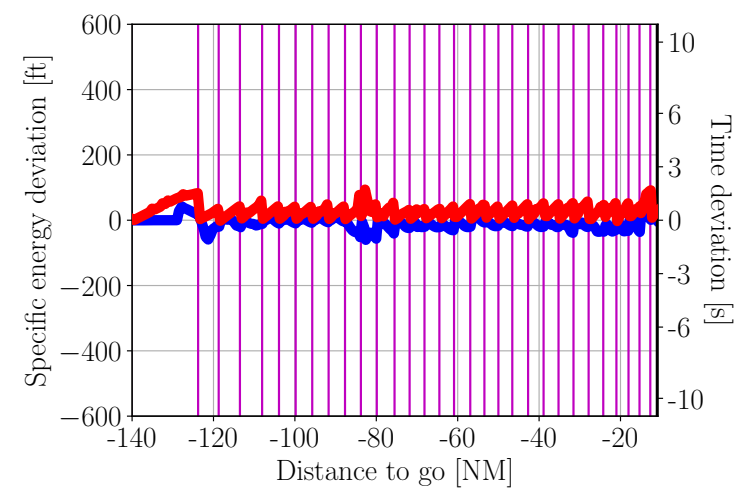

(d) MPC

Fig. 2 Specific energy and time deviations

correct a negative energy deviation, while speed brakes were deployed twice due to an excess of energy.

In strategic guidance the CAS and thrust plans are executed using the elevator and throttle, respectively, and neither time nor energy deviations are corrected as long as they remain within the allowed thresholds. If the energy and/or time error exceed the threshold, the trajectory is re-calculated. Fig. 2(b) shows that five re-plans were triggered by a time error exceeding the upper threshold (i.e., the aircraft was late with respect to the RTA). Whenever a re-plan is triggered, the current state and time are enforced as the initial conditions for the subsequent optimal control problem $\left(\boldsymbol{x}_{0}\right)$, thus both energy and time deviations are nullified at the moment the new plan becomes active.

According to Fig. 3(b), all re-plans required a noticeable faster speed profile if compared with what was initially planned, aiming to correct the accumulated time deviations, which can be as high as the maximum allowed threshold at the distance to go where the re-plan is triggered. Figure 4(b) shows that for none of the re-plans energy modulation with the elevator was sufficient to correct the accumulated time and energy deviations. All re-plans required to add a relatively small amount of energy to the system by means of thrust, aiming to increase the planned speed profile.

In hybrid guidance a tactical controller nullifies sustained time deviations with the elevator while following the thrust plan with the throttle, and energy deviations are corrected by using strategic re-plans. 


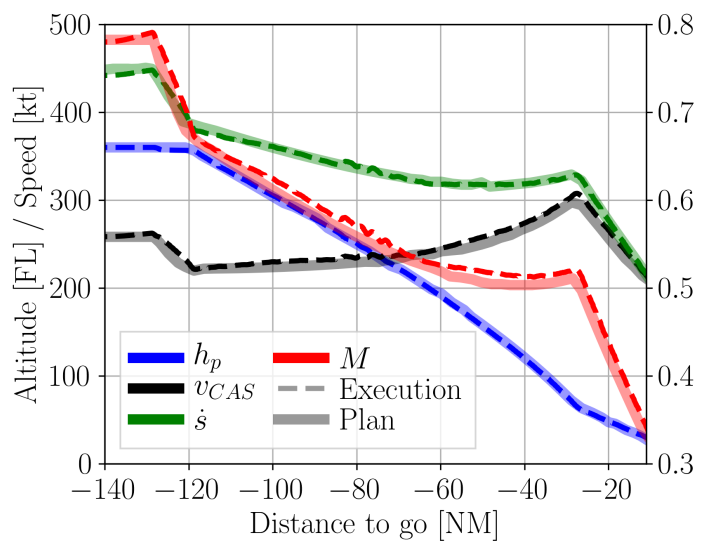

(a) Tactical

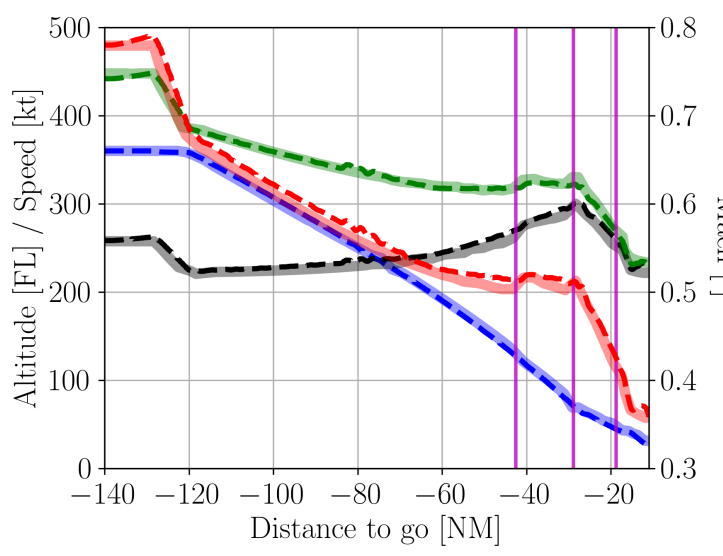

(c) Hybrid

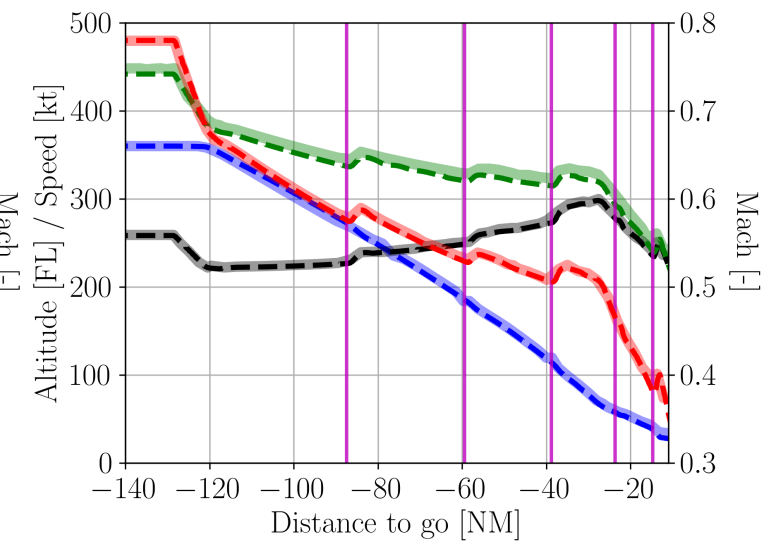

(b) Strategic

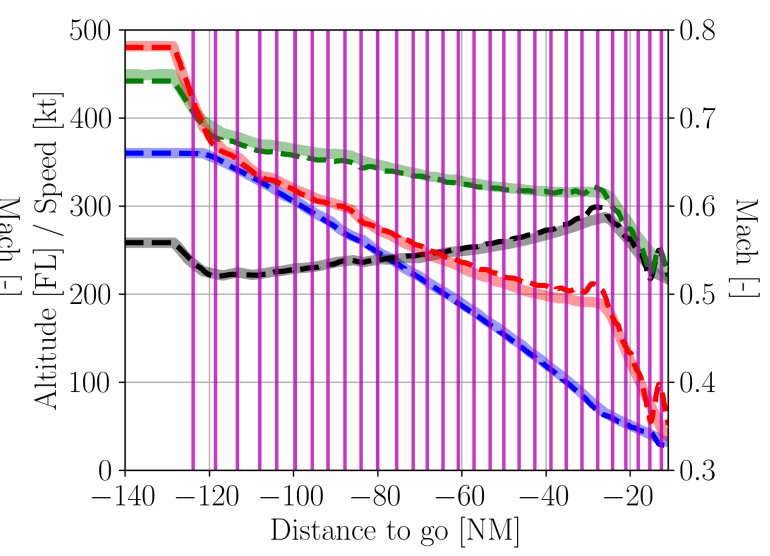

(d) MPC

Fig. 3 Planned and executed states

According to Fig. 3(c) the tactical controller systematically increases the speed above that planned in order to nullify the time deviations caused by the unexpected wind. This higher speed leads to an increase of the aerodynamic drag and, consequently, to the energy loss rate. As a result, three re-plans were triggered in the last 40 NM by an excessive energy deviation reaching the lower threshold (i.e., the aircraft lacked of energy). All re-plans required additional thrust. It should be noted that, since this guidance strategy does not correct energy deviations as long as they remain within the allowed threshold, the metering fix is achieved almost $100 \mathrm{ft}$ below the planned specific total energy level. This missing energy will need to be recovered afterwards by means of additional thrust, leading to more fuel consumption and noise nuisance at low altitudes close to populated areas.

In MPC guidance, energy and time deviations are neither tactically nor strategically corrected during the course of the descent (see Fig. 2(d) . Instead, the trajectory is frequently updated by solving the open-loop optimal control problem described by Eq. (1) over the remaining descent. The aim is to obtain the optimal control vector $\boldsymbol{u}$ that, at each plan update, corrects the actual deviations optimizing the remaining descent for fuel consumption and speed brakes usage. In this particular example, the plan was updated 30 times, reaching SOTIL at the planned time and energy level. 


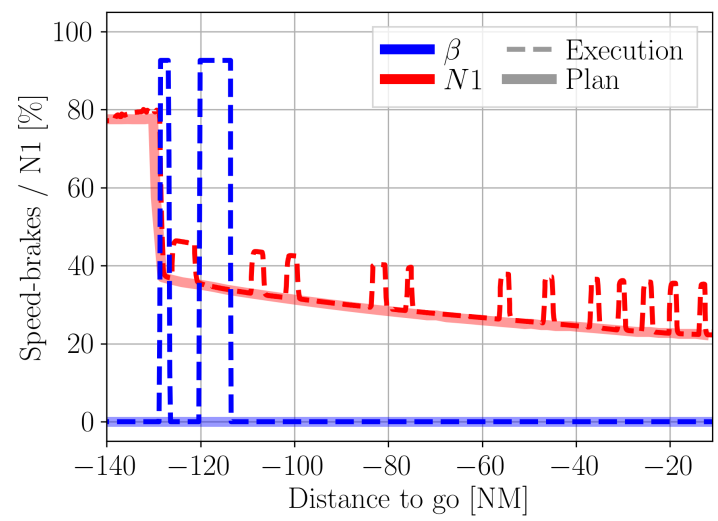

(a) Tactical

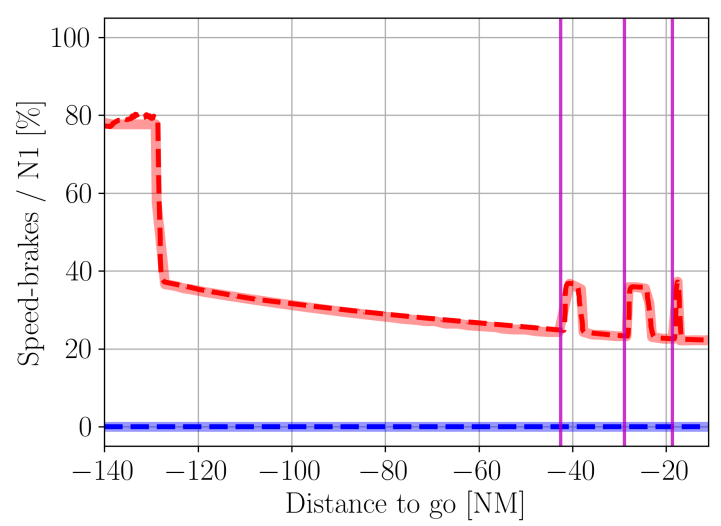

(c) Hybrid

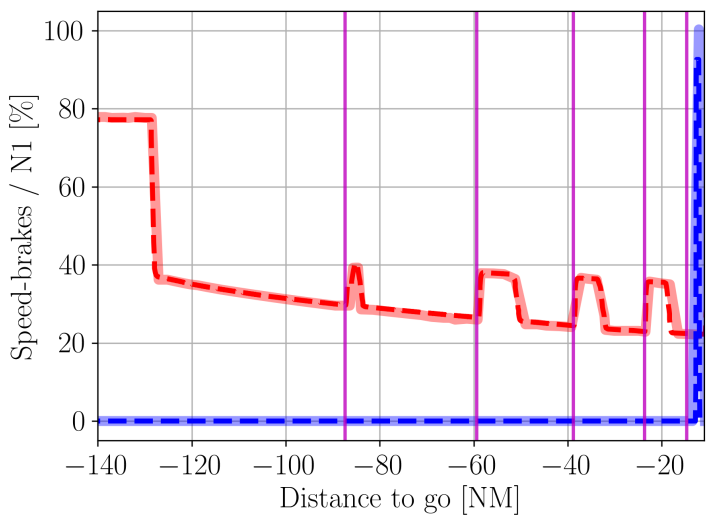

(b) Strategic

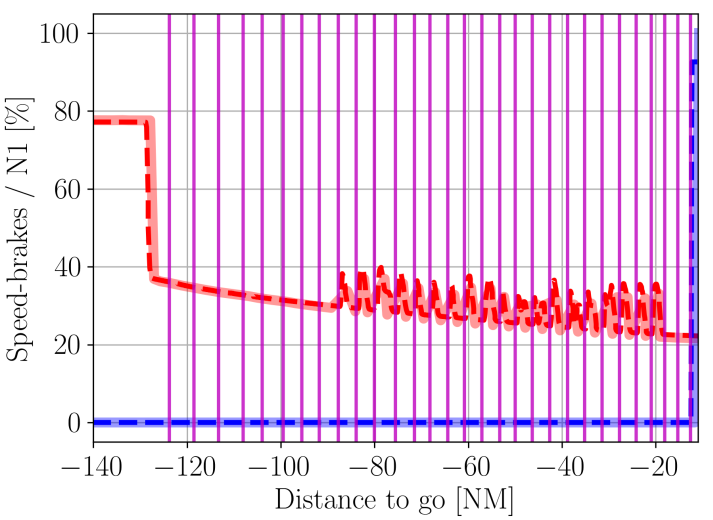

(d) MPC

Fig. 4 Planned and executed controls

According to Fig 4(d), energy modulation was sufficient to correct energy and time deviations until 90 NM from the runway threshold. Thereafter, optimal amounts of energy were added to the system after each re-plan, aiming to correct energy deviations and adjust the speed plan. However, this continuous action of thrust have negative effects including engine wear. If compared with the hybrid and strategic re-plans, MPC re-plans lead to less modifications of the active plan, thus requiring less thrust. The reason behind this fact is that time and energy deviations at the moment the re-plan is triggered are smaller, because they are continuously corrected rather than waiting them to reach a certain threshold.

\section{B. Comparison of guidance strategies}

The comparison of guidance strategies is illustrated using spider charts, which allow to easily identify the trade-offs of each strategy relative to the others. Figure 5(a) gathers the average results from the 492 simulations. For the remaining subfigures, the set of simulations subject to either wind, temperature or aircraft performance model errors were selected separately aiming at isolating their effects.

According to Fig. 5(a), the average time deviation at the metering fix is lower than two seconds for all the guidance strategies, and less than one second for all those different from the strategic. In strategic guidance, time deviations are 


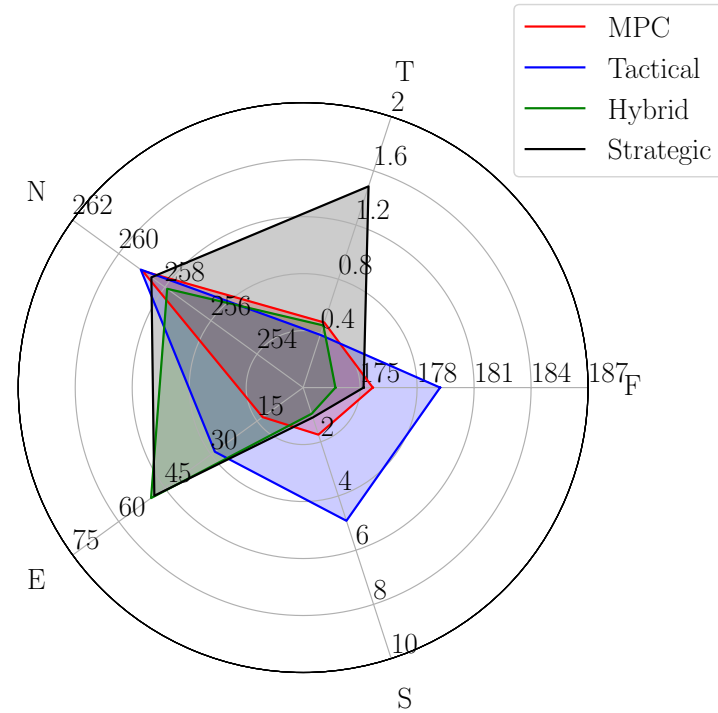

(a) Aggregated results

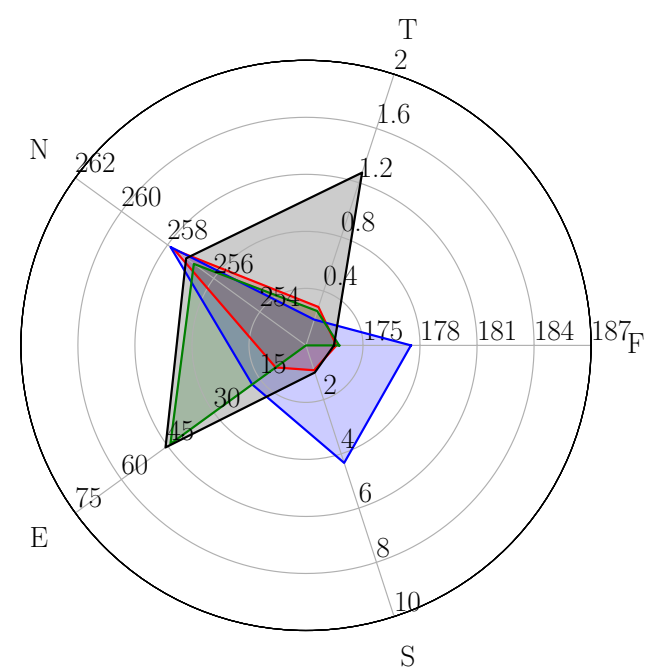

(c) Temperature forecast errors

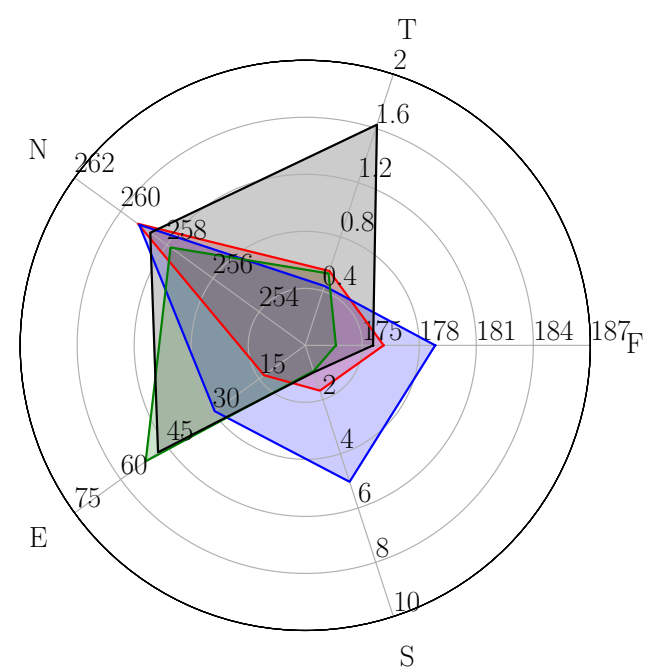

(b) Wind forecast errors

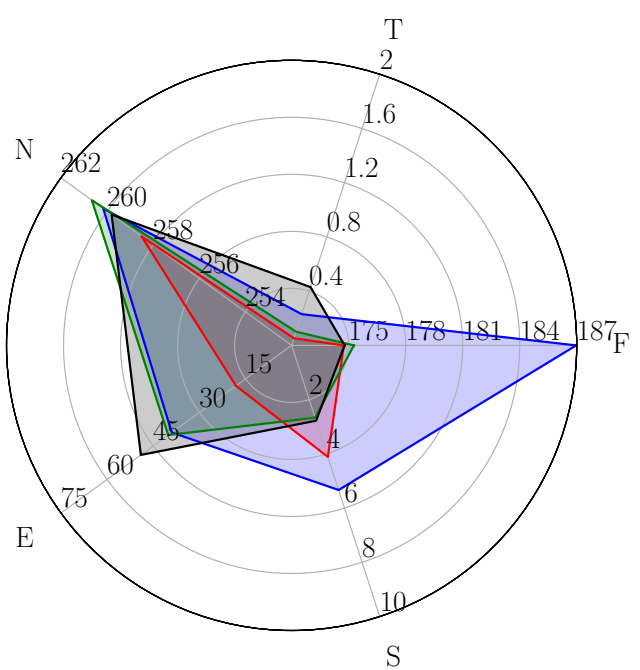

(d) Aircraft performance modelling errors

Fig. 5 Comparison of guidance strategies. E: Specific energy deviation [ft]; F: Fuel consumption [kg]; N: Area affected by aircraft noise $\left[\mathrm{NM}^{2}\right]$; S: Number of speed brakes deployments; T: Time deviation [s].

allowed as long as they remain within the allowed bounds. The remaining strategies show better time deviation figures because their logics continuously correct sustained time deviations, either tactically (in hybrid and tactical) or by means of a continuous re-plan of the optimal descent trajectory (in MPC). These results demonstrate the excellent performance of TEMO to satisfy time constraints.

Regarding the environmental impact, the tactical strategy shows the worst figures in terms of fuel consumption and noise nuisance, due to the continuous (and not optimal) use of throttle and drag devices to correct energy deviations.

The mechanisms used by the MPC are similar to those used by the tactical strategy: energy and time deviations are not monitored, but the elevator, throttle and speed brakes continuously act together to nullify them. However, the 
corrective actions performed by the MPC are mathematically optimal and take into account the remaining descent trajectory. For this reason, the noise nuisance, fuel consumption and speed brakes use are comparable with those of the strategic and hybrid strategies.

The hybrid strategy show the best results in terms of environmental impact mitigation and speed brakes use, proving to exploit the advantages of the strategic and tactical mechanisms. However, the differences with respect to the strategic and MPC strategies are not significant enough to conclude that the hybrid guidance is prefered over the others.

As expected, the specific energy deviation at the metering fix is higher for the strategic and hybrid strategies, which allow deviations up to $100 \mathrm{ft}$ at this fix before triggering a re-plan. Similarly, the tactical strategy permits specific energy deviations up to $50 \mathrm{ft}$ before applying throttle or deploying speed brakes. The MPC shows the best results in terms of energy deviation, because every 30 seconds the energy deviation is nullified when activating the newly computed plan.

It should be noted that the fuel consumption shown in Fig. 5 does not consider the approach and landing phases after the metering fix. If this fix were reached with a certain energy deviation, the missing or excessive energy would need to be added by means of additional thrust (leading to more fuel consumption and noise) or released by means of speed brakes use (leading to more airframe noise nuisance), respectively, at low altitudes and near populated areas.

Using MPC guidance, the aircraft typically reaches the metering fix with negligible energy deviations. Consequently, the MPC guidance does not require the addition nor subtraction of energy after the metering fix.

As expected, and according to Fig. 5(b) wind errors have the effect of increasing both absolute time and energy deviations. It is also worth to note that, in aggregated results (and considering the same number of scenarios with prevalent head wind than with tail wind), the fuel consumption, noise nuisance and speed brakes use are similar to those observed in Fig. 5(a) In addition, Figure 5(c) shows that temperature deviations also lead to significant time and energy deviations. On the one hand, all the guidance strategies considered herein execute the CAS plan, leading to TAS errors in presence of temperature deviations. On the other hand, the aircraft performance (and specially the aircraft thrust) strongly depends on the air temperature. Finally, according to Fig. 5(d), errors in the aircraft performance models are not penalizing the capability to satisfy RTAs, but have a major impact on the environment.

Figure 6 shows, for each guidance strategy, the dependence of each particular metric with respect to the average longitudinal wind speed error $\bar{W}_{s}$. Each point in the scatter plot shows the result of a single simulation. Positive (res. negative) values of $\bar{W}_{s}$ represent unexpected head (resp. tail) wind encountered during the execution of the descent.

According to Fig. 6, the higher the unexpected head wind, the larger the fuel consumption and noise nuisance. In contrast, the number of speed brakes deployments typically increases with the unexpected tail wind. The rationale behind these results is that the head wind leads to an increase of the energy loss rate per unit distance, while the tail wind reduces the amount of energy lost per unit distance. During the execution of the descent, the guidance system will add more energy (directly related to fuel consumption and noise) or will release it by means of speed brakes to safely and efficiently guide the aircraft along the planned trajectory such that the RTA is satisfied. 


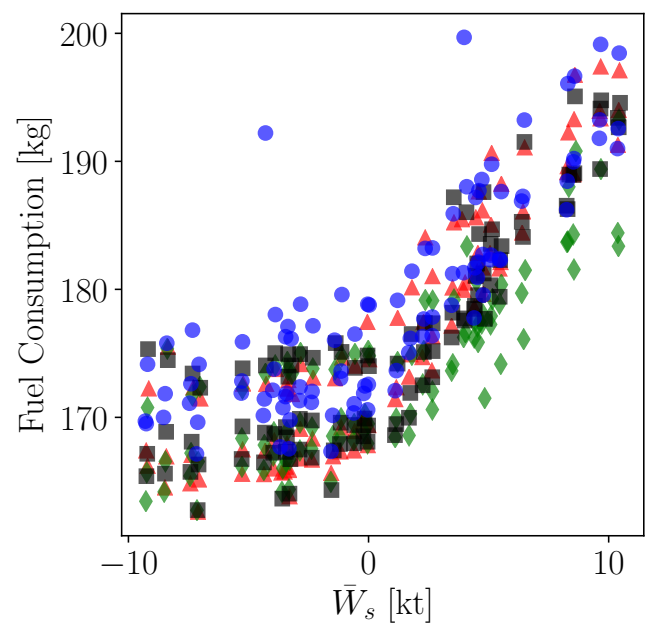

(a) Fuel consumption

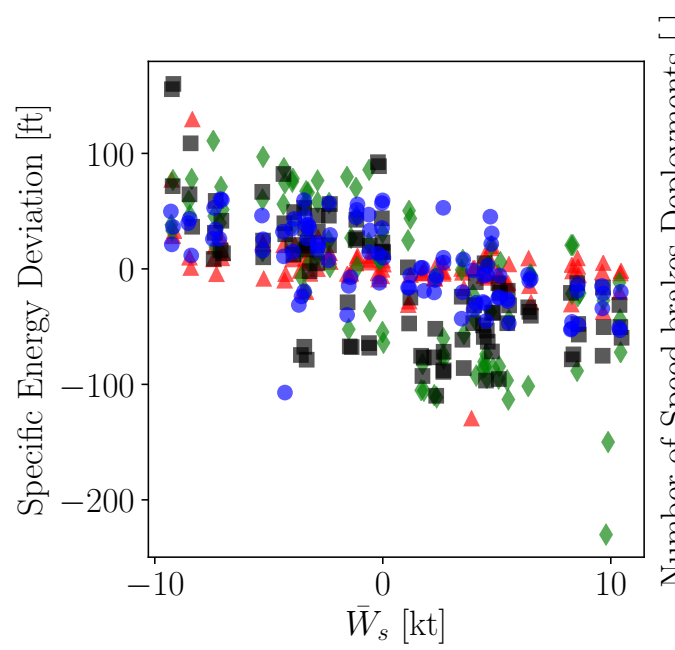

(c) Specific energy deviation

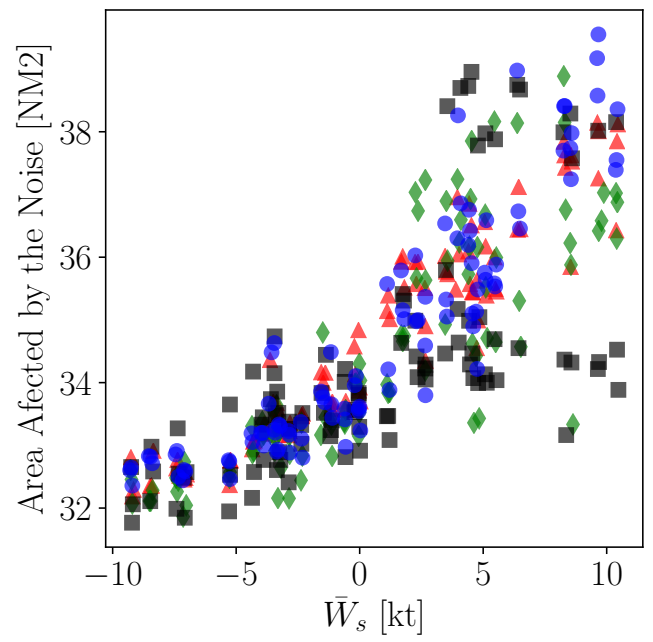

(e) Area afected by the noise

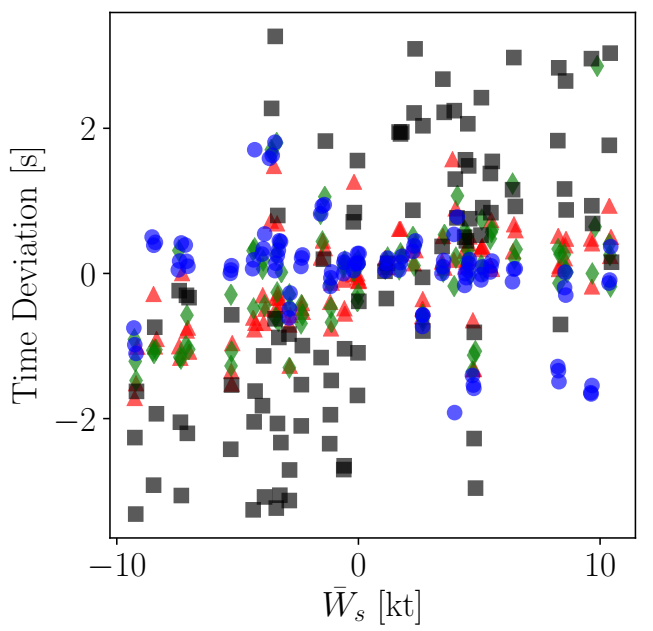

(b) Time deviation

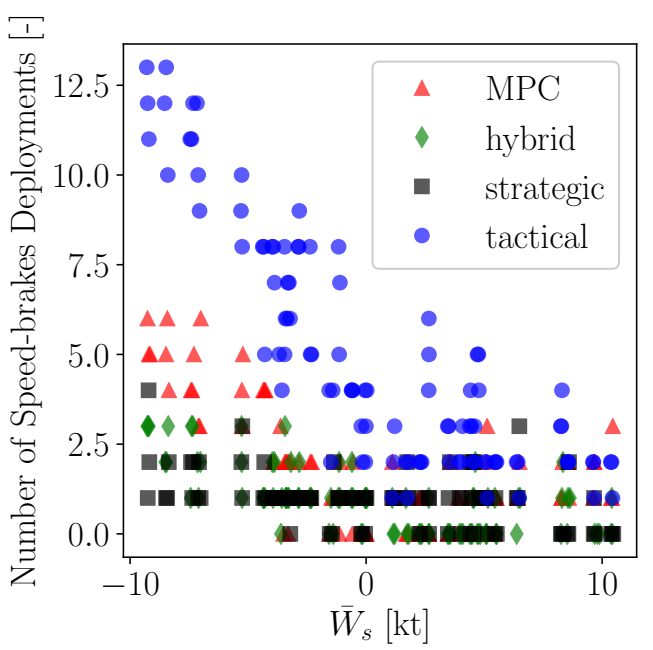

(d) speed brakes use

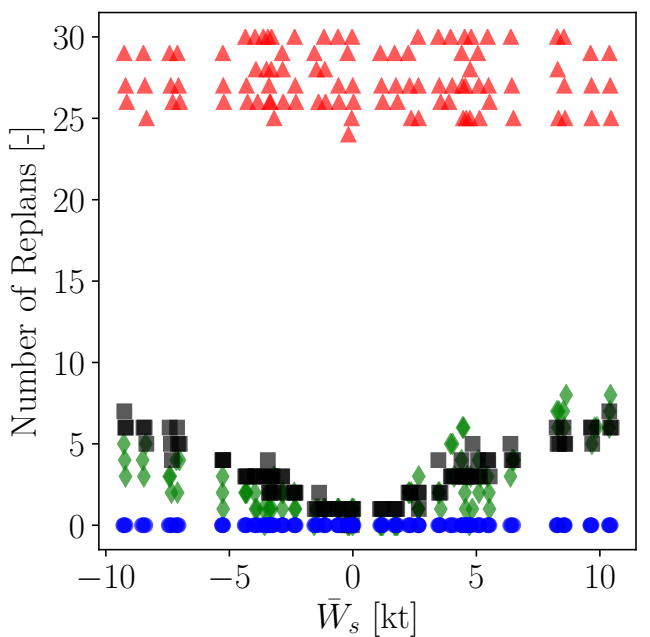

(f) Number of replans

Fig. 6 Correlation between metrics and wind speed error 
According to Figs. 6(c) for the tactical and MPC guidance strategies, the energy deviation at the metering fix is always close to zero, independently of the wind speed error. However, in strategic and hybrid guidance (strategic in the energy channel), more dispersion is found for large wind errors. The same conclusion applies for the time deviation (see Fig. 6(b)]. In this case, the tactical, hybrid and MPC guidance strategies show excellent figures of RTA adherence, while the time deviation in strategic guidance shows a linear correlation with the longitudinal wind speed error.

Figure 6(f) shows that the number of re-plans for the strategic and hybrid guidance strategies strongly depends on the absolute value of the longitudinal wind error. However, the number of re-plans of these guidance strategies is still lower than that of the MPC strategy, which generates a new descent plan starting at the current aircraft state every 30 seconds.

\section{Conclusions}

Results from this batch study demonstrated that all the Time and Energy Management Operations (TEMO) variants can safely accomplish a Required Time of Arrival (RTA) during a Continuous Descent Operation (CDO) with negligible time deviations, even in presence of wind and temperature uncertainties and aircraft performance modeling errors.

The tactical strategy presented herein, which is similar to the guidance strategies used by current Flight Management Systems (FMSs) with the RTA function, requires continuous actions on the throttle and speed brakes deployments to remain on path and arrive on time, leading to more fuel consumption and noise nuisance if compared with other strategies. The hybrid strategy shows the best results in terms of environmental impact mitigation, and also requires less pilot actions to deploy speed brakes. Yet, due to its strategic behavior with respect to energy deviations, the metering fix is reached with higher energy deviations than for other variants. The Model Predictive Control (MPC) approach proved to be the most robust variant regarding energy and time deviations, showing also good environmental impact figures.

It should be noted that all TEMO variants investigated in this note showed very similar results, in terms of environmental impact mitigation and ability to satisfy operational constraints, in presence of errors in the wind forecast and aircraft performance models. However, even these small differences could be significant at aggregated level.

A limitation of the experiment presented herein is that the remaining descent from the metering fix to the runway threshold has not been taken into account. If the energy of the aircraft at this fix is too low, additional thrust would be needed after overflying it. Similarly, if the energy is too high, it would be required to use speed brakes and/or to deploy high-lifting devices or the landing gear earlier.

Last but not least, modern avionics in the most aircraft do not have the computing power to run the 4D trajectory optimization algorithms presented in this note. Electronic Flight Bag (EFB) devices found in most cockpits today, however, might have this potential, but are not allowed to connect directly to the aircraft control systems. Reference [22] showed that this gap could be closed by the pilot operating directly the autopilot, through the Flight Control Unit (FCU), according to guidance advisories given by the TEMO trajectory management algorithms running in the EFB. 


\section{Acknowledgments}

The authors acknowledge the contributions during the preparation of the experiment of Mr. Jaap Groeneweg and Mr. Michiel Valens from NLR. Much appreciation goes out to Mr. Roel Hogenhuis from NLR for his expertise and advice during the noise computation process.

\section{References}

[1] Clarke, J. P. B., Ho, N. T., Ren, L., Brown, J. A., Elmer, K. R., Zou, K. F., Hunting, C., McGregor, D. L., Shivashankara, B. N., Tong, K., Warren, A. W., and Wat, J. K., "Continuous descent approach: Design and flight test for Louisville international airport,” Journal of Aircraft, Vol. 41, No. 5, 2004, pp. 1054-1066. doi:10.2514/1.5572.

[2] Robinson, J., and Kamgarpour, M., "Benefits of Continuous Descent Operations in High-Density Terminal Airspace Under Scheduling Constraints," 10th AIAA Aviation Technology, Integration, and Operations (ATIO), Fort Worth, TX, 2010. doi:10.2514/6.2010-9115.

[3] Itoh, E., Fukushima, S., Hirabayashi, H., Wickramasinghe, N., and Toratani, D., "Evaluating Energy-Saving Arrivals of Wide-Body Passenger Aircraft via Flight-Simulator Experiments," Journal of Aircraft, 2018, pp. 1-17. doi:10.2514/1.C034348.

[4] G. Wu, M., and M. Green, S., "Strategies for Choosing Descent Flight-Path Angles for Small Jets," Journal of Aircraft, Vol. 52 , No. 3, 2012, pp. 847-866. doi:10.2514/6.2012-4817.

[5] De Prins, J. L., Schippers, F. K. M., Mulder, M., Van Paassen, M. M., In ’t Veld, A. C., and Clarke, J.-P., "Enhanced Self-Spacing Algorithm for Three-Degree Decelerating Approaches," Journal of Guidance, Control, and Dynamics, Vol. 30, No. 2, 2007, pp. 576-590. doi:10.2514/1.24542.

[6] Rumbo, J. R., Jackson, M. R., and O’Laughlin, B. E., "Aircraft Control System for Reaching a Waypoint at Required Time of Arrival,”, 2002. Patent No. EP1800197B1, Date of filing: 14/05/2002.

[7] Garrido-Lopez, D., and L. De Prins, J., "Methods and Systems for Aircraft Guidance,”, 2014. Patent No. US2014/0343795A1, Date of filing: 16/05/2014.

[8] Morani, G., Vito, V. D., Corraro, F., Grevtsov, N., and Dymchenko, A., “Automatic Guidance through 4D Waypoints with time and spatial margins," AIAA Guidance, Navigation and Control Conference and Exhibit, Guidance, Navigation, and Control and Co-located Conferences, Boston, MA, 2013. doi:10.2514/6.2013-4892.

[9] Bronsvoort, J., Mcdonald, G., Lopez-leones, J., Vilaplana, M., and Besada, J. A., "Understanding Time-Drift for Different Aircraft Descent Guidance Strategies," International Conference on Application and Theory of Automation in Command and Control Systems (ATACCS), London, UK, 2012, pp. 135-145.

[10] Garrido-López, D., D’Alto, L., and Gómez Ledesma, R., “A novel four-dimensional guidance for continuous descent approaches,” AIAA/IEEE 28th Digital Avionics Systems Conference (DASC), Orlando, FL, 2009. doi:10.1109/DASC.2009.5347433. 
[11] Di Vito, V., Corraro, F., Ciniglio, U., and Verde, L., "An Overview on Systems and Algorithms for On-Board 3D/4D Trajectory Management," Recent Patents on Engineering, Vol. 3, 2009, pp. 149-169. doi:10.2174/187221209789117744.

[12] Klooster, J., Wichman, K., , and Bleeker, O., “4D Trajectory and Time-of-Arrival Control to Enable Continuous Descent Arrivals," AIAA Guidance, Navigation and Control Conference and Exhibit, Guidance, Navigation, and Control and Co-located Conferences, Honolulu, Hawaii, 2008. doi:10.2514/6.2008-7402.

[13] de Jong, P. M. A., Bussink, F. J. L., Verhoeven, R., de Gelder, N., Paassen, M. M. V., and Mulder, M., "Time and Energy Management during Descent and Approach: a human-in-the-loop study," Journal of Aircraft, Vol. 54, No. 1, 2017, pp. 177-189. doi:http://dx.doi.org/10.2514/1.C033741.

[14] de Jong, P. M. A., de Gelder, N., Verhoeven, R., Bussink, F. J. L., Kohrs, R., van Paassen, M. M., and Mulder, M., “Time and Energy Management During Descent and Approach: Batch Simulation Study,” Journal of Aircraft, Vol. 52, No. 1, 2014, pp. 1-14. doi:10.2514/1.C032668.

[15] Bryson, A. E., and Ho, Y., Applied optimal control: optimization, estimation, and control, Taylor and Francis Group, New York, NY, 1975.

[16] Betts, J. T., "Survey of Numerical Methods for Trajectory Optimization,” Journal of Guidance, Control, and Dynamics, Vol. 21, No. 2, 1998, pp. 193-207. doi:10.2514/2.4231.

[17] Dalmau, R., Verhoeven, R., Gelder, N. D., and Prats, X., "Performance comparison between TEMO and a typical FMS in presence of CTA and wind uncertainties," IEEE/AIAA 35th Digital Avionics Systems Conference (DASC), Sacramento, CA, 2016. doi:10.1109/DASC.2016.7778097.

[18] Diehl, M., Bock, H. G., Schlöder, J. P., Findeisen, R., Nagy, Z., and Allgöwer, F., "Real-time optimization and nonlinear model predictive control of processes governed by differential-algebraic equations," Journal of Process Control, Vol. 12, 2002, pp. 577-585. doi:10.1016/S0959-1524(01)00023-3.

[19] Weitz, L. A., and Bai, X., "Using Model Predictive Control for Trajectory Optimization and to Meet Spacing Objectives,” AIAA SciTech Forum. 2018 AIAA Guidance, Navigation, and Control Conference, Kissimmee, FL, 2018. doi:10.2514/6.2018-1599.

[20] Van der Wal, H., Vogel, P., and Wubben, F., "Voorschrift voor de berekening van de lden en lnight geluidbelasting in db(a) ten gevolge van vliegverkeer van en naar de luchtaven.” Tech. rep., National Aerospace Laboratorium (NLR), July 2001.

[21] Robert, E., and Smedt, D. D., "Comparison of operational wind forecasts with recorded flight data," ATM R\&D Seminar (ATM 2013), 2013.

[22] Prats, X., Bendris, B., Dalmau, R., Montolio, J., Day, B., Lenz, H., and Kohrs, R., “4D continuous descent operations supported by an electronic flight bag: A human-in-the-loop study," 2016 IEEE/AIAA 35th Digital Avionics Systems Conference (DASC), 2016, pp. 1-9. doi:10.1109/DASC.2016.7778022. 